\title{
Displaced Non-Keplerian Orbits Using Impulsive Thrust
}

\author{
Colin R McInnes \\ Strathclyde, Glasgow, G1 1XJ, UK \\ Tel: ++44 (0)141548 2049 \\ Fax: ++44 (0)1415525105 \\ colin.mcinnes@strath.ac.uk \\ www.strath.ac.uk/space
}

Advanced Space Concepts Laboratory, Department of Mechanical Engineering, University of

\begin{abstract}
This paper investigates new families of displaced, highly non-Keplerian orbits in the two-body problem and artificial equilibria in the circular restricted three-body problem. The families of orbits presented extend prior work by using periodic impulses to generate displaced orbits rather than continuous thrust. The new displaced orbits comprise a sequence of individual Keplerian arcs whose intersection is continuous in position, with discontinuities in velocity removed using impulses. For frequent impulses the new families of orbits approximate continuous thrust non-Keplerian orbits found in previous studies. To generate approximations to artificial equilibria in the circular restricted three-body problem, periodic impulses are used to generate a sequence of connected three-body arcs which begin and terminate at a fixed position in the rotating frame of reference. Again, these families of orbits reduce to the families of artificial equilibria found using continuous thrust.
\end{abstract}

Keywords Non-Keplerian orbits, circular restricted three-body problem, artificial equilibria, artificial satellites. 


\section{Introduction}

The use of continuous low thrust propulsion to generate artificial equilibria was apparently first proposed by Dusek (1966) who pointed out that a spacecraft could be held at an artificial equilibrium point some distance from a natural three-body equilibrium point. Later, others such as Austin et. al. (1977), Nock (1984) and Yashko and Hastings (1996) noted that propulsive thrust can in principle be used to displace a two-body orbit using continuous thrust normal to the orbit plane. Forward (1984), Baig and McInnes (2008) and Heiligers (2010) have considered displaced geostationary orbits using continuous thrust due to solar radiation pressure. Such displaced two-body orbits will be termed non-Keplerian orbits since the orbit plane does not contain the central mass.

Large families of displaced non-Keplerian two-body orbits have been identified for spacecraft utilising both solar sail (McInnes, 1992a, 1992b) and generic low thrust propulsion (Dankowicz, 1994, McInnes, 1997). More recently displaced orbits have been investigated for a range of applications and propulsion technologies (Mengali and Quarta, 2009, Gong et al, 2009). Studies have determined the conditions for such orbits to exist, their stability properties and their controllability (McInnes, 1997, 1998, Xu and Xu, 2009). Recently, a large catalogue of such orbits was provided by McKay et al (2009) for motion around planetary bodies.

Non-Keplerian two-body orbits are generated using low thrust propulsion by finding conditions for artificial equilibria in a rotating frame of reference. These equilibria then appear as displaced circular orbits when viewed from an inertial frame (McInnes, 1997). Scheeres (1999) considered such hover orbits in a frame of reference rotating with an asteroid. Later, work by Sawai et al (2002) investigated conditions for stability and controllability of such orbits at irregular shaped asteroids.

Conditions for artificial equilibria in the circular restricted three-body problem can be found using solar sails (McInnes et al, 1994), generic low thrust propulsion (Morimoto et al, 2007) and hybrid solar sail propulsion (Baig and McInnes, 2008), while periodic orbits can be found about such artificial equilibria were investigated by Baoyin and McInnes (2006), Waters and McInnes (2007), Baig and McInnes (2009) and Farres and Jorba (2010). Of particular interest is the 
case of low thrust propulsion where regions of stable artificial equilibria were found by Morimoto et al (2007) which do not exist for the solar sail problem. Further investigation of the stability of such artificial equilibria has recently been provided by Bombardelli and Pelaez (2010).

In this paper, new families of displaced non-Keplerian orbits are generated using impulsive, rather than continuous thrust. The use of impulse thrust was considered by Nock (1984), Yashko and Hastings (1996), McInnes (1998), Hope and Trask (2003) and Spilker (2003) to generate small displacements away from a circular Keplerian orbit at linear order in the two-body problem. In this paper, the more general problem is considered with arbitrarily large displacements. The displaced non-Keplerian orbits are approximated by a sequence of individual Keplerian arcs connected by impulses. The sequence of arcs is therefore continuous in position, with discontinuities in velocity which are removed using the impulses. For frequent impulses the families of orbits approximate the continuous thrust non-Keplerian orbits found in previous studies. The conditions for displaced non-Keplerian orbits using impulse thrust can be found by linearising the dynamics of the two and three-body problems in the vicinity of an arbitrary reference point in a rotating frame of reference. While the motion of the spacecraft relative to the reference point is considered at linear order, the reference point can be selected arbitrarily so that, when viewed from an inertial frame, the resulting orbit has arbitrarily large displacements away from a Keplerian orbit. Finally, some applications are considered for displaced geostationary orbits.

\section{Two-Body Non-Keplerian Orbits}

\subsection{Continuous thrust}

Consider the dynamics of a spacecraft at position $\mathbf{r}$ in a frame of reference $R(x, y, z)$ rotating with constant angular velocity $\omega$ relative to an inertial frame $I(X, Y, Z)$, as shown in Fig. 1 (McInnes, 1997, 1998). The spacecraft experiences thrust induced acceleration a and a two-body gravitational potential $V$. The dynamics of the spacecraft $S$ at position $\mathbf{r}=(x, y, z)$ can be defined by an equation of motion in the rotating frame $R$ such that 


$$
\frac{d^{2} \mathbf{r}}{d t^{2}}+2 \boldsymbol{\omega} \times \frac{d \mathbf{r}}{d t}+\boldsymbol{\omega} \times(\boldsymbol{\omega} \times \mathbf{r})=\mathbf{a}-\nabla V(\mathbf{r})
$$

The gravitational potential $V$ of the central body and the thrust-induced acceleration a are defined as

$$
V=-\frac{\mu}{\|\mathbf{r}\|}, \quad \mathbf{a}=\left[\frac{T}{m}\right] \mathbf{n}
$$

where $m$ is the mass of the spacecraft, $\mathbf{n}$ is the direction of the thrust vector and $\mu$ is the two-body gravitational parameter. The condition for equilibrium in the rotating frame of reference $R$ is therefore given by

$$
\mathbf{a}=\nabla V(\mathbf{r})+\boldsymbol{\omega} \times(\boldsymbol{\omega} \times \mathbf{r})
$$

The required thrust magnitude and direction for can then be obtained as

$$
\begin{aligned}
& T(\mathbf{r}, \boldsymbol{\omega})=m\|\nabla V(\mathbf{r})+\boldsymbol{\omega} \times(\boldsymbol{\omega} \times \mathbf{r})\| \\
& \mathbf{n}(\mathbf{r}, \boldsymbol{\omega})=\frac{\nabla V(\mathbf{r})+\boldsymbol{\omega} \times(\boldsymbol{\omega} \times \mathbf{r})}{\|\nabla V(\mathbf{r})+\boldsymbol{\omega} \times(\boldsymbol{\omega} \times \mathbf{r})\|}
\end{aligned}
$$

Using the thrust magnitude and direction defined by Eqs. (4), surfaces of artificial equilibria are generated in the rotating frame of reference, parameterised by the angular velocity of the rotating frame $\omega_{o}=\|\boldsymbol{\omega}\|$. When viewed from an inertial frame of reference an artificial equilibrium point traces a displaced circular orbit of period $2 \pi / \omega_{o}$. Contours of constant thrust induced acceleration generated by Eq. (4a) are shown in Fig. 2. The units are non-dimensionalised with $\mu=1$ so that the unit of acceleration is the gravitational acceleration at unit distance from the 
origin. The angular velocity of the rotating frame of reference is selected as $\omega_{o}=1$ so that a circular Keplerian orbit is found at unit distance in the $X-Y$ plane, as can be seen in the $X-Z$ section shown in Fig. 2. Each point on a contour in Fig. 2 corresponds to a unique displaced circular orbit of period $2 \pi$. In addition, radially displaced orbits are generated at the intersection of the contours with the plane. These orbits exist for orbit radii both greater or less than the circular Keplerian orbit a unit distance from the origin, again with period $2 \pi$. If the spacecraft $S$ is located along the $z$ - axis then it is in static equilibrium with the thrust induced acceleration balancing the local inverse square gravitational acceleration.

Previous studies have investigated families of orbits with different functional forms of the free parameter $\omega_{o}$, their stability properties and their controllability (McInnes, 1997, 1998, Xu and Xu, 2009). It will now be shown that the families of continuous thrust non-Keplerian orbits can be approximated using impulsive thrust by creating a sequence of connected Keplerian arcs, with impulses used to remove discontinuities in velocity at the junction between neighbouring arcs.

\subsection{Impulse thrust}

The analysis of Section 2.1 provides the required thrust magnitude and direction for generic families of continuous thrust non-Keplerian orbits. In this section, the analysis is extended to investigate the use of periodic impulses to generate a sequence of connected Keplerian arcs, which approximate continuous thrust nonKeplerian orbits. The $S$ spacecraft will be considered at some arbitrary position $\mathbf{r}$ in the rotating frame of reference $R$, which can be decomposed into a position $\boldsymbol{\rho}=(\xi, \eta, \zeta)$, relative to an arbitrary point $P$ at position $\tilde{\mathbf{r}}=\left(x_{o}, y_{o}, z_{o}\right)$, as shown in Fig. 1. Viewed from an inertial frame of reference $I$, the point $P$ will trace a displaced circular path. The goal is now to find conditions for a sequence of Keplerian arcs $\rho$, each of which begins and terminates at $P$ with impulses connecting the arcs, as shown in Fig. 3. The point $P$ will therefore trace a displaced non-Keplerian orbit, while the trajectory of the spacecraft $S$ will approximate this displaced orbit by following a sequence of Keplerian arcs.

From Eq. (1), the dynamics of the spacecraft $S$ at position $\mathbf{r}=\tilde{\mathbf{r}}+\boldsymbol{\rho}$ is 
described by

$$
\frac{d^{2}(\tilde{\mathbf{r}}+\boldsymbol{\rho})}{d t^{2}}+2 \boldsymbol{\omega} \times \frac{d(\tilde{\mathbf{r}}+\boldsymbol{\rho})}{d t}+\boldsymbol{\omega} \times(\boldsymbol{\omega} \times(\tilde{\mathbf{r}}+\boldsymbol{\rho}))=-\nabla V(\tilde{\mathbf{r}}+\boldsymbol{\rho})
$$

which can be written as

$$
\frac{d^{2} \boldsymbol{\rho}}{d t^{2}}+2 \boldsymbol{\omega} \times \frac{d \boldsymbol{\rho}}{d t}+\boldsymbol{\omega} \times(\boldsymbol{\omega} \times \boldsymbol{\rho})=-\nabla V(\tilde{\mathbf{r}}+\boldsymbol{\rho})-\boldsymbol{\omega} \times(\boldsymbol{\omega} \times \tilde{\mathbf{r}})
$$

If the displacement $\rho$ of the spacecraft relative to the arbitrary point $P$ at $\tilde{\mathbf{r}}$ is small, then the gradient of the gravitational potential can be expanded to first order as

$$
\nabla V(\tilde{\mathbf{r}}+\boldsymbol{\rho})=\nabla V(\tilde{\mathbf{r}})+\left[\frac{\partial \nabla V}{\partial \mathbf{r}}\right]_{\mathbf{r}=\tilde{\mathbf{r}}} \mathbf{\rho}+\ldots
$$

where the Hessian matrix is evaluated at $P$. The linearised dynamics of the spacecraft $S$ relative to $P$ are now described by

$$
\frac{d^{2} \boldsymbol{\rho}}{d t^{2}}+2 \boldsymbol{\omega} \times \frac{d \boldsymbol{\rho}}{d t}+\boldsymbol{\omega} \times(\boldsymbol{\omega} \times \boldsymbol{\rho})=-\left[\frac{\partial \nabla V}{\partial \mathbf{r}}\right]_{\mathbf{r}=\tilde{\mathbf{r}}} \boldsymbol{\rho}-\nabla V(\tilde{\mathbf{r}})-\boldsymbol{\omega} \times(\boldsymbol{\omega} \times \tilde{\mathbf{r}})
$$

It can be noted that the last two terms of Eq. (8) do not vanish since the point $P$ does not lie on a Keplerian orbit. For the classical linear Clohessey-Wiltshire equations (Clohessey and Wiltshire, 1966), the term $\nabla V(\tilde{\mathbf{r}})+\boldsymbol{\omega} \times(\boldsymbol{\omega} \times \tilde{\mathbf{r}})=0$ on a circular Keplerian reference orbit so that Eq. (8) becomes homogeneous. In addition, the Hessian matrix of the gravitational potential simplifies to diagonal form.

For the displaced non-Keplerian orbit problem considered in this paper, the linear dynamics are non-homogeneous and can be written in compact form as 


$$
\frac{d^{2} \boldsymbol{\rho}}{d t^{2}}=\mathbf{N} \frac{d \mathbf{\rho}}{d t}+\mathbf{M} \boldsymbol{\rho}+\mathbf{Q}
$$

where the skew symmetric gyroscopic matrix $\mathbf{N}$ is given by

$$
\mathbf{N}=\left[\begin{array}{ccc}
0 & 2 \omega_{o} & 0 \\
-2 \omega_{o} & 0 & 0 \\
0 & 0 & 0
\end{array}\right]
$$

and the matrix $\mathbf{M}$ and non-homogeneous part $\mathbf{Q}$ are given by

$$
\begin{aligned}
& \mathbf{M}=\left[\begin{array}{ccc}
V_{x x}+\omega_{o}^{2} & V_{x y} & V_{x z} \\
V_{y x} & V_{y y}+\omega_{o}^{2} & V_{y z} \\
V_{z x} & V_{z y} & V_{z z}
\end{array}\right] \\
& \mathbf{Q}=\left[\begin{array}{c}
Q_{1} \\
Q_{2} \\
Q_{3}
\end{array}\right]
\end{aligned}
$$

Due to the symmetry of the 2-body problem the condition $y_{0}=0$ can be set to simplify the subsequent analysis. However, this constraint is not used in the analysis of the 3-body problem in Section 3 where the cylindrical symmetry of the problem is broken. The components of the Hessian matrix of the gravitational potential are then given by

$$
\begin{aligned}
& V_{x x}=\frac{3 \mu x_{o}^{2}}{\left(x_{o}^{2}+z_{o}^{2}\right)^{5 / 2}}-\frac{\mu}{\left(x_{o}^{2}+z_{o}^{2}\right)^{3 / 2}} \\
& V_{x z}=V_{z x}=\frac{3 \mu x_{o} z_{o}}{\left(x_{o}^{2}+z_{o}^{2}\right)^{5 / 2}}
\end{aligned}
$$




$$
V_{y y}=-\frac{\mu}{\left(x_{o}^{2}+z_{o}^{2}\right)^{3 / 2}}
$$

$$
V_{z z}=\frac{3 \mu z_{o}^{2}}{\left(x_{o}^{2}+z_{o}^{2}\right)^{5 / 2}}-\frac{\mu}{\left(x_{o}^{2}+z_{o}^{2}\right)^{3 / 2}}
$$

where $V_{x y}=V_{y x}=0$ and $V_{y z}=V_{z y}=0$. The non-homogeneous terms are then given by

$$
\begin{gathered}
Q_{1}=\frac{-\mu x_{o}}{\left(x_{o}^{2}+z_{o}^{2}\right)^{3 / 2}}+x_{o} \omega_{o}{ }^{2} \\
Q_{2}=0 \\
Q_{3}=\frac{-\mu z_{o}}{\left(x_{o}^{2}+z_{o}^{2}\right)^{3 / 2}}
\end{gathered}
$$

The resulting system of equations is similar to that considered by Sawai et al (2002) developed for stability and control analysis of a spacecraft hovering at an asteroid.

Defining the system state vector $\mathbf{X}=(\boldsymbol{\rho}, \dot{\boldsymbol{\rho}})^{T}$ for the spacecraft $S$ it can be seen that Eq. (9) can then be written in first order form as

$$
\frac{d \mathbf{X}}{d t}=\mathbf{A X}+\mathbf{B}
$$

where the system matrix $\mathbf{A}$ and non-homogeneous part $\mathbf{B}$ are given by 


$$
\begin{aligned}
& \mathbf{A}=\left[\begin{array}{cccccc}
0 & 0 & 0 & 1 & 0 & 0 \\
0 & 0 & 0 & 0 & 1 & 0 \\
0 & 0 & 0 & 0 & 0 & 1 \\
V_{x x}+\omega_{o}^{2} & 0 & V_{x z} & 0 & 2 \omega_{o} & 0 \\
0 & V_{y y}+\omega_{o}^{2} & 0 & -2 \omega_{o} & 0 & 0 \\
V_{z x} & 0 & V_{z z} & 0 & 0 & 0
\end{array}\right] \\
& \mathbf{B}=\left[\begin{array}{c}
0 \\
0 \\
0 \\
Q_{1} \\
0 \\
Q_{3}
\end{array}\right]
\end{aligned}
$$

In order to obtain a general solution to Eq. (15) it will be necessary to diagonalise the system matrix $\mathbf{A}$. This can be achieved using the eigenvectors $\mathbf{v}_{i}(i=1-6)$ associated with the eigenvalues $\lambda_{\mathrm{i}}(i=1-6)$ of the matrix. The eigenvalues can be determined from the characteristic polynomial of the system matrix $\mathbf{A}$ from $\operatorname{det}\|\mathbf{A X}-\lambda \mathbf{I}\|=0$ in the usual manner. The characteristic polynomial then reduces to a bi-cubic equation of the form

$$
\eta^{3}+K_{1} \eta^{2}+K_{2} \eta+K_{3}=0
$$

where $\eta=\lambda^{2}$ and

$$
\begin{aligned}
& K_{1}=2 \omega_{o}^{2} \\
& K_{2}=\omega_{o}^{4}-\frac{6 \mu^{2}}{\left(x_{o}^{2}+z_{o}^{2}\right)^{2}}+\frac{3 \mu^{2}}{\left(x_{o}^{2}+z_{o}^{2}\right)^{3}}+\frac{3 \mu \omega_{o}^{2}\left(x_{o}^{2}-2 z_{o}^{2}\right)}{\left(x_{o}^{2}+z_{o}^{2}\right)^{5 / 2}}
\end{aligned}
$$




$$
\begin{aligned}
K_{3}= & \frac{3 \mu^{2} \omega_{o}^{2}\left(x_{o}^{2}+2 z_{o}^{2}\right)}{\left(x_{o}^{2}+z_{o}^{2}\right)^{4}}-\frac{2 \mu^{2} \omega_{o}^{2}}{\left(x_{o}^{2}+z_{o}^{2}\right)^{3}}-\frac{2 \mu^{3}}{\left(x_{o}^{2}+z_{o}^{2}\right)^{9 / 2}} \\
& -\frac{3 \mu \omega_{o}^{4} z_{o}^{2}}{\left(x_{o}^{2}+z_{o}^{2}\right)^{5 / 2}}+\frac{\mu \omega_{o}^{4}}{\left(x_{o}^{2}+z_{o}^{2}\right)^{3 / 2}}
\end{aligned}
$$

In general, the eigenvalue spectrum obtained from Eq. (18) consists of two pairs of conjugate imaginary eigenvalues and a pair of real eigenvalues. The eigenvectors $\mathbf{v}_{\mathrm{i}}$ corresponding to each eigenvalue $\lambda_{\mathrm{i}}$ can be used to assemble a matrix $\mathbf{V}=\left[\mathbf{v}_{1}, \ldots \mathbf{v}_{i}, \ldots \mathbf{v}_{6}\right]$ from the eigenvectors which can then be used to diagonalise the system matrix A (Betounes, 2010). The diagonal form of the system matrix $\mathbf{A}$ is then defined by $\mathbf{D}=\mathbf{V}^{-\mathbf{1}} \mathbf{A V}$ where

$$
\mathbf{D}=\left[\begin{array}{llll}
\lambda_{1} & & & \\
& \cdot & & \\
& & \cdot & \\
& & \lambda_{6}
\end{array}\right]
$$

Defining a new state vector $\mathbf{U}=\mathbf{V}^{-1} \mathbf{X}$ it can be seen that Eq. (15) can be transformed to

$$
\frac{d \mathbf{U}}{d t}=\left(\mathbf{V}^{-1} \mathbf{A} \mathbf{V}\right) \mathbf{U}+\mathbf{V}^{-1} \mathbf{B}
$$

Finally, defining a matrix $\mathbf{E}$ as

$$
\mathbf{E}=\left[\begin{array}{lll}
\exp \left(\lambda_{1} t\right) & & \\
& \cdot & \\
& \cdot & \\
& & \exp \left(\lambda_{6} t\right)
\end{array}\right]
$$


it can be shown that Eq. (21) can be integrated directly and so the general solution to the inhomogeneous linear system defined by Eq. (15) is given by

$$
\mathbf{X}(t)=\mathbf{V}(\mathbf{E}-\mathbf{I}) \mathbf{D}^{-1} \mathbf{V}^{-1} \mathbf{B}+\mathbf{V} \mathbf{E}^{-1} \mathbf{V X}(0)
$$

where $\mathbf{X}(0)$ is a set of arbitrary initial conditions.

This general solution can then be more usefully written in terms of an effective state transition matrix $\boldsymbol{\Phi}(t)=\mathbf{V E}^{-1} \mathbf{V}$ as

$$
\mathbf{X}(t)=\mathbf{\Phi}(t) \mathbf{X}(0)+\Gamma
$$

where $\boldsymbol{\Gamma}=\mathbf{V}(\mathbf{E}-\mathbf{I}) \mathbf{D}^{-1} \mathbf{V}^{-1} \mathbf{B}$. Decomposing the state transition matrix and nonhomogeneous part as

$$
\begin{aligned}
& \boldsymbol{\Phi}(t)=\left[\begin{array}{ll}
\boldsymbol{\Phi}_{11}(t) & \boldsymbol{\Phi}_{12}(t) \\
\boldsymbol{\Phi}_{21}(t) & \boldsymbol{\Phi}_{22}(t)
\end{array}\right] \\
& \boldsymbol{\Gamma}=\left[\begin{array}{l}
\boldsymbol{\Gamma}_{1} \\
\boldsymbol{\Gamma}_{2}
\end{array}\right]
\end{aligned}
$$

the dynamics of the spacecraft $S$ relative to the point $P$ are defined by

$$
\left[\begin{array}{c}
\boldsymbol{\rho}(t) \\
\dot{\boldsymbol{\rho}}(t)
\end{array}\right]=\left[\begin{array}{ll}
\boldsymbol{\Phi}_{11}(t) & \boldsymbol{\Phi}_{12}(t) \\
\boldsymbol{\Phi}_{21}(t) & \boldsymbol{\Phi}_{22}(t)
\end{array}\right]\left[\begin{array}{l}
\boldsymbol{\rho}(0) \\
\dot{\boldsymbol{\rho}}(0)
\end{array}\right]+\left[\begin{array}{c}
\boldsymbol{\Gamma}_{1} \\
\boldsymbol{\Gamma}_{2}
\end{array}\right]
$$

which provides the general solution to Eq. (8). The trajectory of $S$ relative to $P$ is therefore given by

$$
\boldsymbol{\rho}(t)=\boldsymbol{\Phi}_{11}(t) \boldsymbol{\rho}(0)+\boldsymbol{\Phi}_{12}(t) \dot{\boldsymbol{\rho}}(0)+\boldsymbol{\Gamma}_{1}
$$


In order to generate a displaced non-Keplerian orbit the condition is set that $\boldsymbol{\rho}(\tau)=\boldsymbol{\rho}(0)$, where $\tau$ is the duration of the Keplerian arc described by Eq. (27). This condition ensures that a sequence of connected Keplerian arcs are generated which are continuous in position, but discontinuous in velocity. As will be seen later $\tau$ can be selected as a simple fraction of the orbit period of point $P$. Since the point $P$ is arbitrary, the initial conditions of $S$ can be selected such that $\boldsymbol{\rho}(0)=0$. From Eq. (26) the required initial velocity to ensure that $\boldsymbol{\rho}(\tau)=\boldsymbol{\rho}(0)$ is then given by

$$
\dot{\boldsymbol{\rho}}(0)=-\boldsymbol{\Phi}_{12}^{-1}(\tau) \boldsymbol{\Gamma}_{1}
$$

The velocity of $S$ on return to $P$ after time $\tau$ is then

$$
\dot{\boldsymbol{\rho}}(\tau)=\boldsymbol{\Phi}_{22}(\tau) \dot{\boldsymbol{\rho}}(0)+\boldsymbol{\Gamma}_{2}
$$

and so the impulse required to connect neighbouring Keplerian arcs is given by

$$
\Delta \mathbf{v}=\left[\boldsymbol{\Phi}_{22}(\tau) \boldsymbol{\Phi}_{12}^{-1}(\tau)-\boldsymbol{\Phi}_{12}^{-1}(\tau)\right] \boldsymbol{\Gamma}_{1}-\boldsymbol{\Gamma}_{2}
$$

as shown schematically in Fig. 3.

In order to illustrate the use of impulses to generate families of displaced non-Keplerian orbits, the family of continuous thrust orbits shown in Fig. 2 will be considered. Again, the angular velocity of the rotating frame of reference is selected as $\omega_{o}=1$, so that a circular Keplerian orbit will found at unit distance in the plane. The reference point $P$ in the rotating frame will be selected as $\tilde{\mathbf{r}}=(0.5,0,0.5)$. The non-Keplerian orbit will therefore have a radius and displacement distance of 0.5 in non-dimensional units, as shown in Fig. 4. In order to generate a sequence of Keplerian arcs which connect to form the displaced orbit, the duration of each arc is selected as a simple fraction of the orbit period $\tau=2 \pi / \omega_{0} N$. Here $N=10$ is selected for illustration. Using the condition 
$\boldsymbol{\rho}(\tau)=\boldsymbol{\rho}(0)$ a sequence of 10 arcs are generated, where each arc returns to the point $P$ in the rotating frame, as can be seen in Fig. 4. The arcs are generated using Eq. (27), the linear solution to Eq. (8), with the impulses connecting the Keplerian arcs defined by Eq. (30).

In order to evaluate the accuracy of the linear approximation, the twopoint boundary value problem defined by $\boldsymbol{\rho}(\tau)=\boldsymbol{\rho}(0)$ can be solved numerically using a shooting method for the full non-linear dynamics defined by Eq. (6). Fig. 5 shows the components of the displacement $\boldsymbol{\rho}=(\xi, \eta, \zeta)$ of the spacecraft $S$ from the point $P$. It can be seen that the linear solution provides a good approximation to the full non-linear dynamics of the problem in this case. Cleary for large displacements about a planetary body, the required impulse magnitude will be extremely large. However, Section 4 provides an example of a displaced geostationary orbit where the impulse magnitude is modest.

\subsection{Reduction to Clohessy-Wiltshire equations}

As the number of Keplerian arcs increases it is expected that non-Keplerian orbits using continuous and impulse thrust will match asymptotically. To demonstrate this, the linearised dynamics at an arbitrary point $\mathrm{P}$ can be reduced to the Clohessy-Wiltshire equations (Clohessey and Wiltshire, 1966). For $y_{o}=z_{o}=0$ the system matrix $\mathbf{A}$ and non-homogeneous part $\mathbf{B}$ from Eqs. (16-17) reduce to

$$
\mathbf{A}=\left[\begin{array}{cccccc}
0 & 0 & 0 & 1 & 0 & 0 \\
0 & 0 & 0 & 0 & 1 & 0 \\
0 & 0 & 0 & 0 & 0 & 1 \\
\frac{2 \mu}{x_{o}^{3}}+\omega_{o}^{2} & 0 & 0 & 0 & 2 \omega_{o} & 0 \\
0 & -\frac{\mu}{x_{o}^{3}}+\omega_{o}^{2} & 0 & -2 \omega_{o} & 0 & 0 \\
0 & 0 & -\frac{\mu}{x_{o}^{3}} & 0 & 0 & 0
\end{array}\right]
$$

and 


$$
\mathbf{B}=\left[\begin{array}{c}
0 \\
0 \\
0 \\
-\frac{\mu}{x_{o}^{2}}+\omega_{o}^{2} \\
0 \\
0
\end{array}\right]
$$

However, if $P$ is then defined on a circular Keplerian orbit of radius $x_{0}$ then $\omega_{o}^{2}=\mu / x_{o}^{3}$ and so Eq. (8) reduces to

$$
\begin{aligned}
& \ddot{\xi}-2 \omega_{o} \dot{\eta}-3 \omega_{o}^{2} \xi=a_{\xi} \\
& \ddot{\eta}+2 \omega_{o} \dot{\xi}=a_{\eta} \\
& \ddot{\zeta}+\omega_{o}^{2} \zeta=a_{\zeta}
\end{aligned}
$$

where $\left(a_{\xi}, a_{\eta}, a_{\zeta}\right)$ correspond to the components of continuous thrust induced acceleration a in the local frame attached to $P$. The continuous thrust acceleration is re-introduced to allow a comparison of continuous and impulse thrust nonKeplerian orbits. For continuous thrust, the required acceleration components for an equilibrium solution at some point $\boldsymbol{\rho}=\left(\xi_{o}, 0, \zeta_{o}\right)$ in the rotating frame of reference can be obtained from Eqs. (33) as

$$
\begin{aligned}
& a_{\xi}=-3 \omega_{o}^{2} \xi_{o} \\
& a_{\eta}=0 \\
& a_{\zeta}=\omega_{o}^{2} \zeta_{o}
\end{aligned}
$$


which can also be obtained from Eq. (3) for small displacements from a circular Keplerian orbit in the plane.

If there is no thrust induced acceleration, then the Clohessy-Wiltshire equations Eq. (33) have a general solution (Clohessy and Wiltshire, 1966) of the form

$$
\begin{aligned}
\xi(t)= & {\left[\frac{\dot{\xi}_{o}}{\omega_{o}}\right] \sin \left(\omega_{o} t\right)-\left[3 \xi_{o}+\frac{2 \dot{\eta}_{o}}{\omega_{o}}\right] \cos \left(\omega_{o} t\right)+\left[\frac{2 \dot{\eta}_{o}}{\omega_{o}}+4 \xi_{o}\right] } \\
\eta(t)= & {\left[\frac{4 \dot{\eta}_{o}}{\omega_{o}}+6 \xi_{o}\right] \sin \left(\omega_{o} t\right)+\left[\frac{2 \dot{\xi}_{o}}{\omega_{o}}\right] \cos \left(\omega_{o} t\right) } \\
& -\left[3 \dot{\eta}_{o}+6 \omega_{o} \xi_{o}\right] t+\left[\xi_{o}-\frac{2 \dot{\xi}_{o}}{\omega_{o}}\right] \\
\zeta(t)= & \zeta_{o} \cos \left(\omega_{o} t\right)+\frac{\dot{\zeta}_{o}}{\omega_{o}} \sin \left(\omega_{o} t\right)
\end{aligned}
$$

A displaced non-Keplerian orbit can then be obtained using periodic impulses, as discussed in Section 2.2. To maintain an out-of-plane displacement, repeated vertical impulses are required such that $\zeta(\tau)=\zeta_{o}$ where $\tau$ is again the period between impulses (McInnes, 1998). Then, using Eq. (35c), the required out-ofplane velocity at the start of the Keplerian arc is given by

$$
\dot{\zeta}_{o}=\omega_{o} \zeta_{o} \tan \left[\frac{\omega_{o} \tau}{2}\right]
$$


Due to the symmetry of the problem it can be seen that $\dot{\zeta}(T)=-\dot{\zeta}(0)$ and so the effective out-of-plane acceleration $\bar{a}_{\zeta}$ provided by the repeated impulses is then $2 \dot{\zeta}(0) / \tau$ such that

$$
\bar{a}_{\zeta}=\frac{2 \omega_{o} \zeta_{o}}{\tau} \tan \left[\frac{\omega_{o} \tau}{2}\right]
$$

If the time $\tau$ between impulses is small, Eq. (37) can then be expanded to yield

$$
\bar{a}_{\zeta}=\omega_{o}^{2} \zeta_{o}+\frac{1}{12} \omega_{o}^{4} \zeta_{o} \tau^{2}+\ldots
$$

Comparing Eq. (38) and Eq. (34c) it can be seen that to first order the required accelerations are equal, with the higher order terms representing the difference in required mean acceleration between the continuous thrust and impulse cases.

For a radial displacement, the condition $\eta(0)=0$ will be used along with the requirements $\eta(\tau)=0$ and $\xi(\tau)=\xi_{o}$. Again using Eqs. (35) it can be seen that the required initial velocity components are given by

$$
\begin{gathered}
\dot{\xi}(0)=\frac{3 \omega_{o}^{2} \tau \xi_{o} \sin \left(\omega_{o} \tau / 2\right)}{3 \omega_{o} \tau \cos \left(\omega_{o} \tau / 2\right)-8 \sin \left(\omega_{o} \tau / 2\right)} \\
\dot{\eta}(0)=-6 \omega_{o} \xi_{o} \frac{\omega_{o} \tau \cos \left(\omega_{o} \tau / 2\right)-2 \sin \left(\omega_{o} \tau / 2\right)}{3 \omega_{o} \tau \cos \left(\omega_{o} \tau / 2\right)-8 \sin \left(\omega_{o} \tau / 2\right)}
\end{gathered}
$$

However, it is found that $\dot{\eta}(\tau)=\dot{\eta}(0)$ and $\dot{\xi}(\tau)=-\dot{\xi}(0)$ so that only repeated radial impulses are required (McInnes, 1998). The effective radial acceleration $\bar{a}_{x}$ provided by the repeated impulses is then $2 \dot{\xi}(0) / \tau$. Expanding Eq. (39a) for small periods $\tau$ it is found that 


$$
\bar{a}_{\xi}=-3 \omega_{o}^{2} \xi_{o}+\frac{3}{4} \omega_{o}^{4} \xi_{o} \tau^{2}+\ldots
$$

Again, comparing Eq. (40) and Eq. (34a) it can be seen that to first order the required accelerations are equal, again with the higher order terms representing the difference in mean acceleration between the continuous thrust and impulse cases. These approximations provide a simple means of assessing the required acceleration and accumulated effective $\Delta v$ for small displacements in the vicinity of a Keplerian orbit, along with the difference in cost of the continuous and impulse thrust modes of operation.

\section{Three-Body Non-Keplerian Orbits}

\subsection{Continuous thrust}

The analysis provided in Section 2 can be extended to consider artificial equilibria in the circular restricted three-body problem. Again, artificial equilibria can be generated using continuous low thrust or periodic impulses to connect a sequence of 3-body ballistic arcs. The analysis for the 3-body problem is similar to that provided in Section 2. Here, the 3-body gravitational potential is defined as

$$
V=-\frac{(1-\tilde{\mu})}{\left\|\mathbf{r}_{1}\right\|}-\frac{\tilde{\mu}}{\left\|\mathbf{r}_{2}\right\|}
$$

where the position of the spacecraft $S$ relative to the two primary masses $m_{1}$ and $m_{2}$ is defined by $\mathbf{r}_{1}=(x+\tilde{\mu}, y, z)$ and $\mathbf{r}_{1}=(1-\tilde{\mu}+x, y, z)$, as shown in Fig. 6 . The

mass ratio of the problem is defined by $\tilde{\mu}=m_{2} /\left(m_{1}+m_{2}\right)$ where the gravitational constant and the sum of the primary masses are taken to be unity. Unlike the families of 2-body orbits, the angular velocity of the rotating frame of reference is defined uniquely by the orbital angular velocity of the primary masses and so is taken to be unity. 
Using this new potential, Eq. (4) can then provide the required thrust magnitude and direction for equilibria in the rotating frame of reference, as used by Morimoto et al (2007). Contours of equal thrust induced acceleration are shown in Fig. 7 for a mass ratio of $\tilde{\mu}=3.04 \times 10^{-6}$ (Earth-Sun system). The condition $\nabla V(\mathbf{r})+\boldsymbol{\omega} \times(\boldsymbol{\omega} \times \mathbf{r})=0$ can be seen at the $\mathrm{L}_{1}$ and $\mathrm{L}_{2}$ natural equilibrium points where $\mathbf{a}=0$. For a modest thrust induced acceleration, nested surfaces of artificial equilibria are generated in the vicinity of the natural equilibrium points.

\subsection{Impulse thrust}

Again, the procedure detailed in Section 2.2 can be followed to define the requirements for periodic motion near artificial equilibria using impulsive thrust. Periodic impulses are now used to generate a sequence of connected ballistic three-body arcs, which approximate continuous thrust artificial equilibria. The spacecraft $S$ will again be considered at some position $\mathbf{r}$ in the rotating frame of reference, which can be decomposed into a position $\boldsymbol{\rho}=(\xi, \eta, \zeta)$, relative to an arbitrary point $P$ at position $\tilde{\mathbf{r}}=\left(x_{o}, y_{o}, z_{o}\right)$, as shown in Fig. 6. If conditions can be found for the spacecraft $S$ to return to $P$ then an approximation can be found to the families of artificial equilibria using continuous thrust. The goal is therefore to find conditions for a sequence of ballistic three-body $\operatorname{arcs} \rho$, each of which begins and terminates at $P$ with impulses connecting the arcs. The spacecraft $S$ will therefore remain in the vicinity of $\mathrm{P}$.

Using Eq. (41), the components of the Hessian matrix of the 3-body gravitational potential defined by Eq. (11) are found to be

$$
\begin{aligned}
V_{x x}= & \frac{3 \tilde{\mu}\left(-1+\tilde{\mu}+x_{o}\right)^{2}}{\left(\left(-1+\tilde{\mu}+x_{o}\right)^{2}+y_{o}^{2}+z_{o}^{2}\right)^{5 / 2}}-\frac{\tilde{\mu}}{\left(\left(-1+\tilde{\mu}+x_{o}\right)^{2}+y_{o}^{2}+z_{o}^{2}\right)^{3 / 2}} \\
& -\frac{3(-1+\tilde{\mu})\left(\tilde{\mu}+x_{o}\right)^{2}}{\left(\left(\tilde{\mu}+x_{o}\right)^{2}+y_{o}^{2}+z_{o}^{2}\right)^{5 / 2}}+\frac{-1+\tilde{\mu}}{\left(\left(\tilde{\mu}+x_{o}\right)^{2}+y_{o}^{2}+z_{o}^{2}\right)^{3 / 2}} \\
V_{x y}= & \frac{3 \tilde{\mu}\left(-1+\tilde{\mu}+x_{o}\right) y_{o}}{\left(\left(-1+\tilde{\mu}+x_{o}\right)^{2}+y_{o}^{2}+z_{o}^{2}\right)^{5 / 2}}-\frac{3(-1+\tilde{\mu})\left(\tilde{\mu}+x_{o}\right) y_{o}}{\left(\left(\tilde{\mu}+x_{o}\right)^{2}+y_{o}^{2}+z_{o}^{2}\right)^{5 / 2}}
\end{aligned}
$$




$$
\begin{aligned}
V_{x z} & =\frac{3 \tilde{\mu}\left(-1+\tilde{\mu}+x_{o}\right) z_{o}}{\left(\left(-1+\tilde{\mu}+x_{o}\right)^{2}+y_{o}^{2}+z_{o}^{2}\right)^{5 / 2}}-\frac{3(-1+\tilde{\mu})\left(\tilde{\mu}+x_{o}\right) z_{o}}{\left(\left(\tilde{\mu}+x_{o}\right)^{2}+y_{o}^{2}+z_{o}^{2}\right)^{5 / 2}} \\
V_{y x} & =\frac{3 \tilde{\mu}\left(-1+\tilde{\mu}+x_{o}\right) y_{o}}{\left(\left(-1+\tilde{\mu}+x_{o}\right)^{2}+y_{o}^{2}+z_{o}^{2}\right)^{5 / 2}}-\frac{3(-1+\tilde{\mu})\left(\tilde{\mu}+x_{o}\right) y_{o}}{\left(\left(\tilde{\mu}+x_{o}\right)^{2}+y_{o}^{2}+z_{o}^{2}\right)^{5 / 2}} \\
V_{y y} & =\frac{3 \tilde{\mu} y_{o}^{2}}{\left(\left(-1+\tilde{\mu}+x_{o}\right)^{2}+y_{o}^{2}+z_{o}^{2}\right)^{5 / 2}}-\frac{\tilde{\mu}}{\left(\left(-1+\tilde{\mu}+x_{o}\right)^{2}+y_{o}^{2}+z_{o}^{2}\right)^{3 / 2}} \\
& -\frac{3(-1+\tilde{\mu}) y_{o}^{2}}{\left(\left(\tilde{\mu}+x_{o}\right)^{2}+y_{o}^{2}+z_{o}^{2}\right)^{5 / 2}}+\frac{-1+\tilde{\mu}}{\left(\left(\tilde{\mu}+x_{o}\right)^{2}+y_{o}^{2}+z_{o}^{2}\right)^{3 / 2}}
\end{aligned}
$$

$$
\begin{aligned}
& V_{y z}=\frac{3 \tilde{\mu} y_{o} z_{o}}{\left(\left(-1+\tilde{\mu}+x_{o}\right)^{2}+y_{o}^{2}+z_{o}^{2}\right)^{5 / 2}}-\frac{3(-1+\tilde{\mu}) y_{o} z_{o}}{\left(\left(\tilde{\mu}+x_{o}\right)^{2}+y_{o}^{2}+z_{o}^{2}\right)^{5 / 2}} \\
& V_{z x}=\frac{3\left(-1+\tilde{\mu}+x_{o}\right) z_{o}}{\left(\left(-1+\tilde{\mu}+x_{o}\right)^{2}+y_{o}^{2}+z_{o}^{2}\right)^{5 / 2}}-\frac{3(-1+\tilde{\mu})\left(\tilde{\mu}+x_{o}\right) z_{o}}{\left(\left(\tilde{\mu}+x_{o}\right)^{2}+y_{o}^{2}+z_{o}^{2}\right)^{5 / 2}}
\end{aligned}
$$

$$
V_{z y}=\frac{3 \tilde{\mu} y_{o} z_{o}}{\left(\left(-1+\tilde{\mu}+x_{o}\right)^{2}+y_{o}^{2}+z_{o}^{2}\right)^{5 / 2}}-\frac{3(-1+\tilde{\mu}) y_{o} z_{o}}{\left(\left(\tilde{\mu}+x_{o}\right)^{2}+y_{o}^{2}+z_{o}^{2}\right)^{5 / 2}}
$$

$$
\begin{aligned}
V_{z z} & =\frac{3 \tilde{\mu} z_{o}^{2}}{\left(\left(-1+\tilde{\mu}+x_{o}\right)^{2}+y_{o}^{2}+z_{o}^{2}\right)^{5 / 2}}-\frac{\tilde{\mu}}{\left(\left(-1+\tilde{\mu}+x_{o}\right)^{2}+y_{o}^{2}+z_{o}^{2}\right)^{3 / 2}} \\
& -\frac{3(-1+\tilde{\mu}) z_{o}^{2}}{\left(\left(\tilde{\mu}+x_{o}\right)^{2}+y_{o}^{2}+z_{o}^{2}\right)^{5 / 2}}+\frac{-1+\tilde{\mu}}{\left(\left(\tilde{\mu}+x_{o}\right)^{2}+y_{o}^{2}+z_{o}^{2}\right)^{3 / 2}}
\end{aligned}
$$

while the non-homogeneous terms are given by 


$$
\begin{aligned}
& Q_{1}=\frac{\tilde{\mu}\left(-1+\tilde{\mu}+x_{o}\right)}{\left(\left(-1+\tilde{\mu}+x_{o}\right)^{2}+y_{o}^{2}+z_{o}^{2}\right)^{5 / 2}}+\frac{(-1+\tilde{\mu})\left(\tilde{\mu}+x_{o}\right)}{\left(\left(\tilde{\mu}+x_{o}\right)^{2}+y_{o}^{2}+z_{o}^{2}\right)^{5 / 2}}+x_{o} \\
& Q_{2}=\frac{\tilde{\mu} y_{o}}{\left(\left(-1+\tilde{\mu}+x_{o}\right)^{2}+y_{o}^{2}+z_{o}^{2}\right)^{5 / 2}}+\frac{(-1+\tilde{\mu}) y_{o}}{\left(\left(\tilde{\mu}+x_{o}\right)^{2}+y_{o}^{2}+z_{o}^{2}\right)^{5 / 2}}+y_{o} \\
& Q_{3}=\frac{\tilde{\mu} z_{o}}{\left(\left(-1+\tilde{\mu}+x_{o}\right)^{2}+y_{o}^{2}+z_{o}^{2}\right)^{5 / 2}}+\frac{(-1+\tilde{\mu}) z_{o}}{\left(\left(\tilde{\mu}+x_{o}\right)^{2}+y_{o}^{2}+z_{o}^{2}\right)^{5 / 2}}
\end{aligned}
$$

Then, the conditions for the spacecraft $S$ to return to the point $P$ are again defined by Eq. (30) using the matrix entries defined by Eq. (42) and (43).

In order to illustrate the use of impulses to generate families of artificial 3body equilibria, the family of continuous thrust equilibria shown in Fig. 7 will be considered. Eight reference points $P_{1-8}$ in the rotating frame will be selected at $\tilde{\mathbf{r}}=(0.99, \pm 0.005, \pm 0.005)$ and $\tilde{\mathbf{r}}=(1.01, \pm 0.005, \pm 0.005)$ in the vicinity of the natural $\mathrm{L}_{1}$ and $\mathrm{L}_{2}$ points. In order to generate a sequence of ballistic 3-body arcs which return to the point $P$, the duration of each arc is again selected as a simple fraction $N$ of the orbit period of the two primary masses. Here $N=5$ is selected for illustration to generate large arcs (where $N=10$ was used in Section 2.2). Using the condition $\boldsymbol{\rho}(\tau)=\boldsymbol{\rho}(0)$ a sequence of arcs are generated, where each arc returns to each point $P$ in the rotating frame, as can be seen in Fig. 8. The arcs are again generated using Eq. (26), the linear solution to Eq. (8) with the potential defined in Eq. (41) and the impulses connecting the arcs defined by Eq. (30). For the example provided in Fig. 8 the impulse magnitude is $1082.2 \mathrm{~ms}^{-1}$ for the EarthSun system. Clearly this is large, but is more modest for points closer to the natural equilibria.

Lastly, in order to evaluate the accuracy of the linear approximation, the two-point boundary value problem defined by $\boldsymbol{\rho}(\tau)=\boldsymbol{\rho}(0)$ can be solved numerically using a shooting method for the full non-linear dynamics. Fig. 9 shows the components of the displacement $\boldsymbol{\rho}=(\xi, \eta, \zeta)$ of the spacecraft $S$ from the point $P$. It can be seen that the linear solution provides a reasonable approximation to the full non-linear dynamics of the problem. However, the match is poorer than the 2-body problem due to the large trajectory loops generated by using a smaller number of arcs with $N=5$, rather than $N=10$. A larger 
number of arcs will generate a smaller displacement of $S$ from $P$ and so better accuracy for the linear solution.

\section{Applications}

Highly non-Keplerian orbits have a range of potential application, as discussed in Section 1. Here, an example is provided for a displaced geostationary orbit. Geostationary spacecraft typically use a station-keeping box sized at 0.05 deg of orbit arc. This corresponds to a physical size of order 35x35 km. Figure 10 shows a displaced orbit with a displacement distance of $35 \mathrm{~km}$ using both continuous thrust and periodic impulses $(N=10)$. Applications of such displaced orbits include stacking geostationary satellite at crowded longitudes in a similar manner to the solar sail displaced orbits considered by Forward (1984) and later Baig and McInnes (2010) and Heiligers (2010). The required impulse magnitude for the displaced orbit shown in Fig. 10 can be determined from Eq. (30) as $1.654 \mathrm{~ms}^{-1}$, so that the $\Delta v$ per orbit is $16.54 \mathrm{~ms}^{-1}$. The required continuous thrust induced acceleration can be determined from Eq. (4a) as $1.851 \times 10^{-4} \mathrm{~ms}^{-2}$, so that the effective $\Delta v$ per orbit for the continuous thrust case is $15.99 \mathrm{~ms}^{-1}$. There is therefore a modest penalty of order $0.5 \mathrm{~ms}^{-1}$ for using the impulse mode rather than the continuous thrust mode. In addition, continuous low thrust allows the use of high specific impulse propulsion, such as an electric thruster with a specific impulse of $3000 \mathrm{~s}$, compared to a bi-propellant thruster with a specific impulse of 320 s used for the impulse mode (Heiligers, 2010). For a large $4000 \mathrm{~kg}$ geostationary platform, the propellant mass per orbit for the continuous thrust case is $2.15 \mathrm{~kg}$, which rises to $21.02 \mathrm{~kg}$ for the impulse mode.

\section{Conclusions}

Families of displaced 2-body non-Keplerian orbits and 3-body artificial equilibria have been extended by considering impulse rather than continuous thrust. The displaced non-Keplerian orbits comprise a sequence of individual Keplerian arcs connected by impulses. The sequence of arcs are therefore continuous in position, with discontinuities in velocity which are removed using the impulses. For frequent impulses it has been shown that the new families of orbits approximate 
continuous thrust non-Keplerian orbits. The conditions for displaced nonKeplerian orbits using impulse thrust were found by linearising the dynamics of the two and three-body problems in the vicinity of an arbitrary reference point in a rotating frame. While the motion of the spacecraft relative to the reference point was considered at linear order, the reference point was be selected arbitrarily so that when viewed from an inertial frame of reference the non-Keplerian orbit has arbitrarily large displacements.

\section{Acknowledgments}

The work presented in this paper was funded through European Research Grant 227571 VISIONSPACE.

\section{References}

Austin, R.E., Dod, R.E. and Terwilliger, C.H.: The ubiquitous solar electric propulsion stage. Acta Astronaut. 4(5-6), 671-694 (1977)

Baoyin, H. and McInnes, C.R.: Solar sail halo orbits at the Sun-Earth artificial $\mathrm{L}_{1}$ point. Celest. Mech. Dyn. Astro 94(2), 155-171 (2006)

Baig, S., and McInnes, C. R.: Artificial three-body equilibria for hybrid low-thrust propulsion. J. Guid. Control, and Dyn. 31(6), 1644-1655 (2008)

Baig, S. and McInnes, C.R.: Artificial halo orbits for low-thrust propulsion spacecraft. Celest. Mech. Dyn. Astro 104(4), 321-335 (2009)

Baig, S., and McInnes, C.R.: Light-levitated geostationary orbits are feasible. J. Guid. Control, and Dyn. 33(3), 782-793 (2010)

Betounes, D.: Differential equations: theory and applications, Springer, New York, 201-207 (2010)

Bombardelli, C., and Pelaez, J.: On the stability of artificial equilibrium points in the circular restricted three-body problem. Celest. Mech. Dyn. Astro. On-line 15 October 2010, DOI: 10.1007/s10569-010-9317-z

Clohessy, W.H., and Wiltshire, R.S.: Terminal Guidance for Satellite Rendezvous. J. Aerosp. Sci. 27(9), 653-678 (1996)

Dankowicz, H.: Some special orbits in the two-body problem with radiation pressure. Celest. Mech. Dyn. Astro. 58(4), 353-370 (1994)

Dusek, H. M.: Motion in the vicinity of libration points of a generalized restricted three-body model. Prog. Astronaut. Aeronaut. 17, 37-54 (1966) 
Farres, A., and Jorba, A.: Periodic and quasi-periodic motions of a solar sail close to $\mathrm{SL}_{1}$ in the Earth-Sun system. Celest. Mech. Dyn. Astro. 107(1-2), 233-253 (2010)

Forward, R. L.: Light levitated geostationary cylindrical orbits using perforated light sails. J. Astronaut. Sci. 32(2), 221-226 (1984)

Gong, S., Li, J., and Baoyin, H.: Formation flying solar sail gravity tractors in displaced orbit for towing near-Earth asteroids. Celest. Mech. Dyn. Astro 105(13), 159-177 (2009)

Heiligers, J.: Displaced geostationary orbits using hybrid low-thrust propulsion. 61st Int. Astronaut. Cong. IAC.10.E2.1.2. Prague, Czech Republic, 27 Sept - 1 Oct, 2010

Hope, A., and Trask, A.: Pulsed thrust method for hover formation flying. Adv. Astronaut. Sci. 116, 2423-2433 (2003)

McInnes, C. R., and Simmons, J. F. L.: Solar sail halo orbits I: Heliocentric case. J. Spacecr. Rockets. 29(4), 466-471 (1992a)

McInnes, C. R., and Simmons, J. F. L.: Solar sail halo orbits II: Geocentric case. J. Spacecr. Rockets 29(4), 472-479 (1992b)

McInnes, C. R., McDonald, A. J. C., Simmons, J. F. L., and MacDonald, E.: Solar sail parking in restricted three-body systems. J. Guid. Control, and Dyn. 17(2), 399-406 (1994)

McInnes, C. R.: The existence and stability of families of displaced two-body orbits. Celest. Mech. Dyn. Astro. 67(2), 167-180 (1997)

McInnes, C. R.: Dynamics, stability, and control of displaced non-Keplerian orbits. J. Guid. Control, and Dyn. 21(5), 799-805 (1998)

McKay, R. J., Macdonald, M., Bosquillon de Frescheville, F., Vasile, M., McInnes, C. R., and Biggs, J. D.: Non-Keplerian orbits using low thrust, high IsP propulsion systems. $60^{\text {th }}$ Int. Astronaut. Cong. IAC.09.C1.2.8, Daejeon, Republic of Korea, Oct $12^{\text {th }}-16^{\text {th }}, 2009$

Mengali, G., and Quarta, AA.: Non-Keplerian orbits for electric sails. Celest. Mech. Dyn. Astro 105(1-3), 179-195 (2009)

Morimoto, M. Y., Yamakawa, H., and Uesugi, K.: Artificial equilibrium points in the low-thrust restricted three-body problem. J. Guid. Control, and Dyn. 30(5), 1563-1567 (2007)

Nock, K. T.: Rendezvous with Saturn's rings. Anneaux des Planetes, IAU Coll. No. 75, Cepadues Editions, Toulouse, 743-759 (1984)

Sawai, S., Scheeres, D. J., and Broschart, S.: Control of hovering spacecraft using altimetry. J. Guid. Control, and Dyn. 25(4), 786-795 (2002) 
Scheeres, D.J.: Stability of hovering orbits around small bodies. Adv. Astro. Sci. 102, 855-875 (1999)

Spilker, T.: Saturn ring observer. Acta. Astro. 52(2-6), 259-265 (2003)

Waters, T., and McInnes, C.R.: Periodic orbits above the ecliptic plane in the solar sail restricted 3-body problem. J. Guid. Control, and Dyn. 30(3), 687-693 (2007)

$\mathrm{Xu}, \mathrm{M}$., and $\mathrm{Xu}, \mathrm{S} .:$ Nonlinear dynamical analysis for displaced orbits above a planet. Celest. Mech. Dyn. Astro. 102(4), 327-353 (2009)

Yashko, G.J., and Hastings, D.E.: Analysis of thruster requirements and capabilities for local satellite clusters. $10^{\text {th }}$ AIAA/USU Small Satellite Conf. Logan, Utah, Sept 16-19, 1996. 


\section{Figure Captions}

Figure 1. Frame $R$ rotating with angular velocity $\omega$ relative to inertial frame $I$. Reference point $P$ at position $\widetilde{\mathbf{r}}$ and spacecraft $S$ at position $\rho$ relative $P$.

Figure 2. Contours of non-dimensional thrust induced acceleration for artificial equilibria in rotating frame $R$ (2-body problem).

Figure 3. Sequence of Keplerian arcs which begin and terminate at $P$ connected by impulses (2-body problem).

Figure 4. Displaced orbits constructed from sequence of Keplerian arcs; Orbit I $\tilde{\mathbf{r}}=(0.5,0.0,0.5)$, Orbit II $\tilde{\mathbf{r}}=(0.75,0.0,0.75)$, Orbit III $\tilde{\mathbf{r}}=(1.0,0.0,1.0)$. Dashed lined indicates equivalent constant acceleration displaced orbit.

Figure 5. Comparison of linear and non-linear solutions for Orbit I. Solid line linear solution, dashed line non-linear solution.

Figure 6. Frame $R$ rotating with angular velocity $\|\boldsymbol{\omega}\|=1$ relative to inertial frame $I$. Reference point $P$ at position $\tilde{\mathbf{r}}$ and spacecraft $S$ at position $\rho$ relative $P$.

Figure 7. Contours of non-dimensional thrust induced acceleration for artificial equilibria in rotating frame $R$ (3-body problem) for $\tilde{\mu}=3.04 \times 10^{-6}$.

Figure 8. Artificial 3-body equilibria constructed from sequences of 3-body arcs; $P_{1} \tilde{\mathbf{r}}=(0.991,0,0.005), P_{2} \tilde{\mathbf{r}}=(0.991,0,-0.005), P_{3} \tilde{\mathbf{r}}=(0.991,0.005,0.0)$, $P_{4} \tilde{\mathbf{r}}=(0.991,-0.005,0.0), P_{5} \tilde{\mathbf{r}}=(1.011,0,0.005), P_{6} \tilde{\mathbf{r}}=(1.011,0,-0.005)$, $P_{7} \tilde{\mathbf{r}}=(1.011,0.005,0.0), P_{8} \tilde{\mathbf{r}}=(1.011,-0.005,0.0)$.

Figure 9. Comparison of linear and non-linear solutions for $P_{1}$. Solid line linear solution, dashed line non-linear solution.

Figure 10. Displaced two-body geostationary orbit (35 km displacement). 
Figure 1.

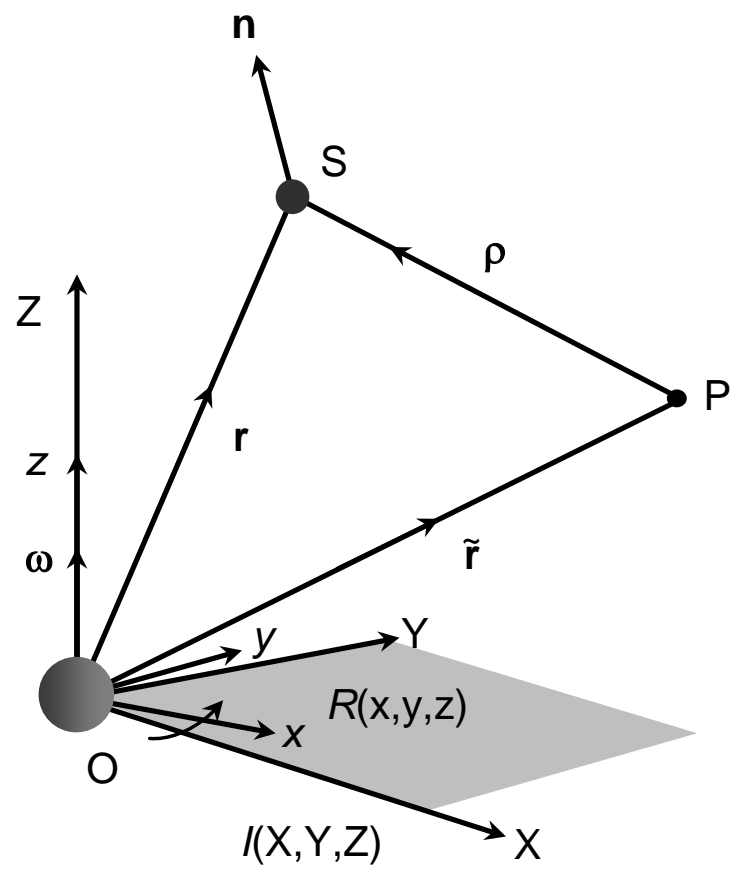


Figure 2.

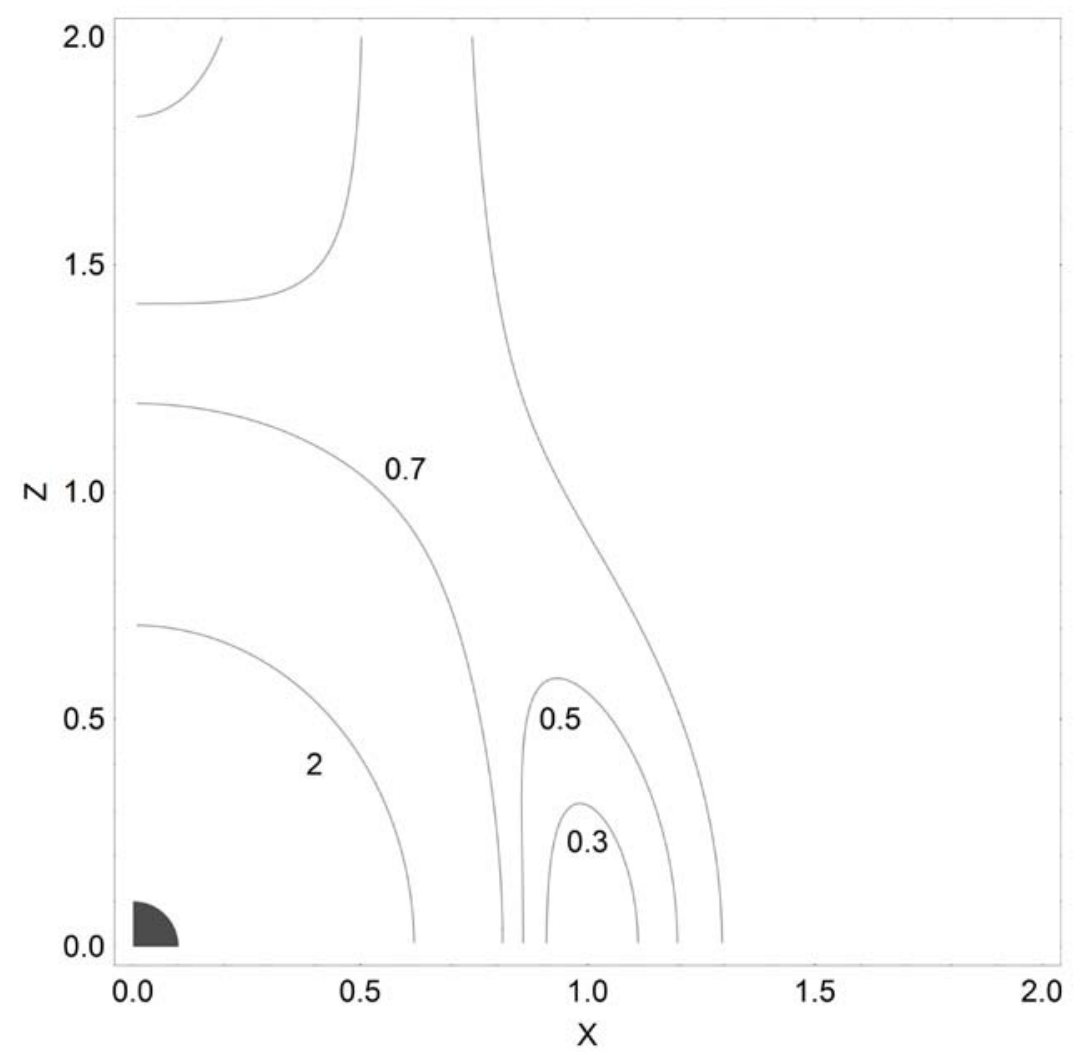


Figure 3.

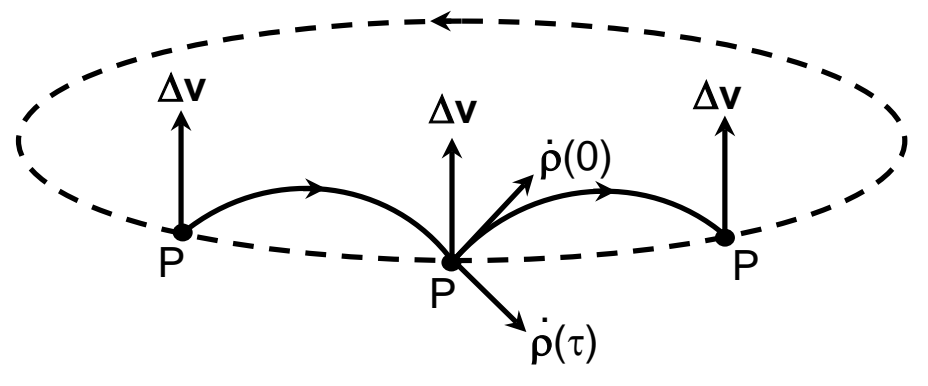


Figure 4.

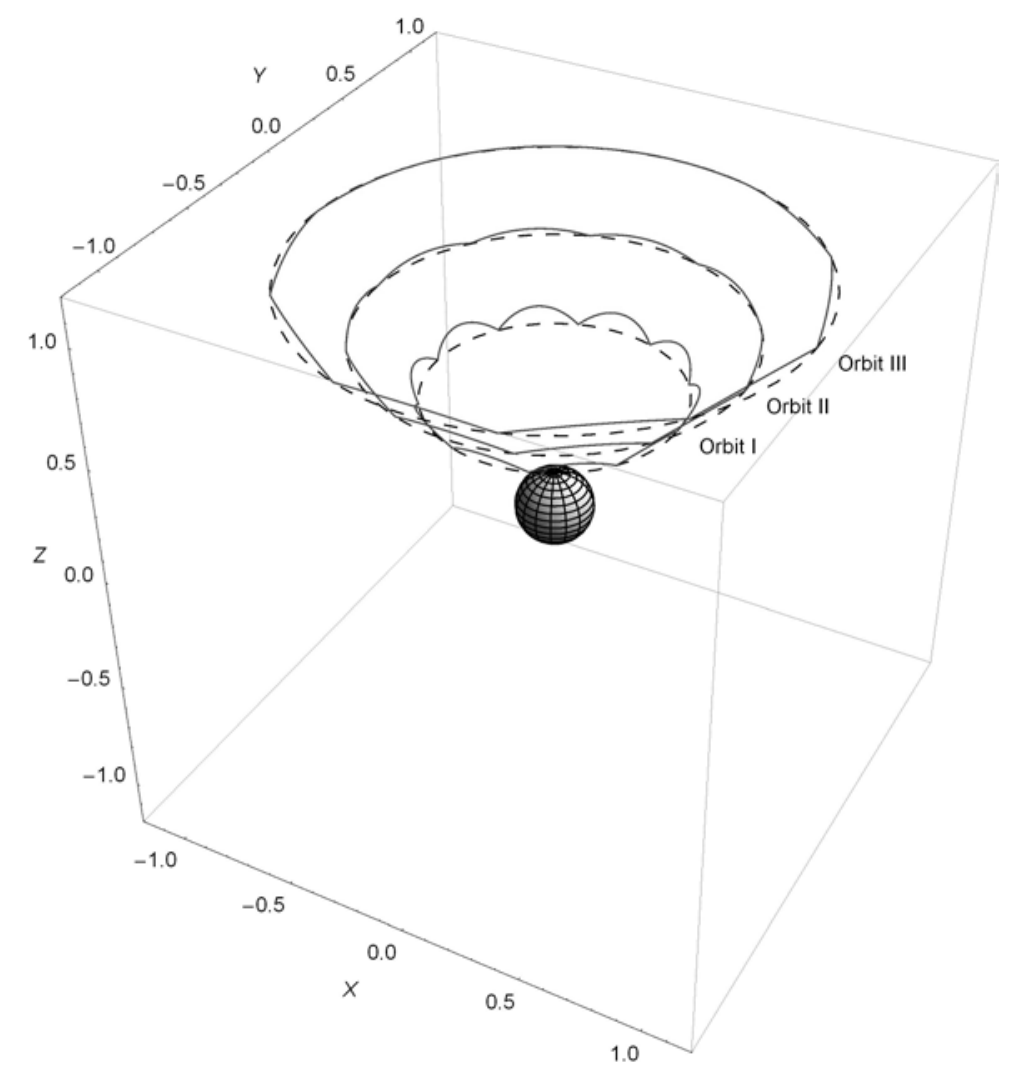


Figure 5.

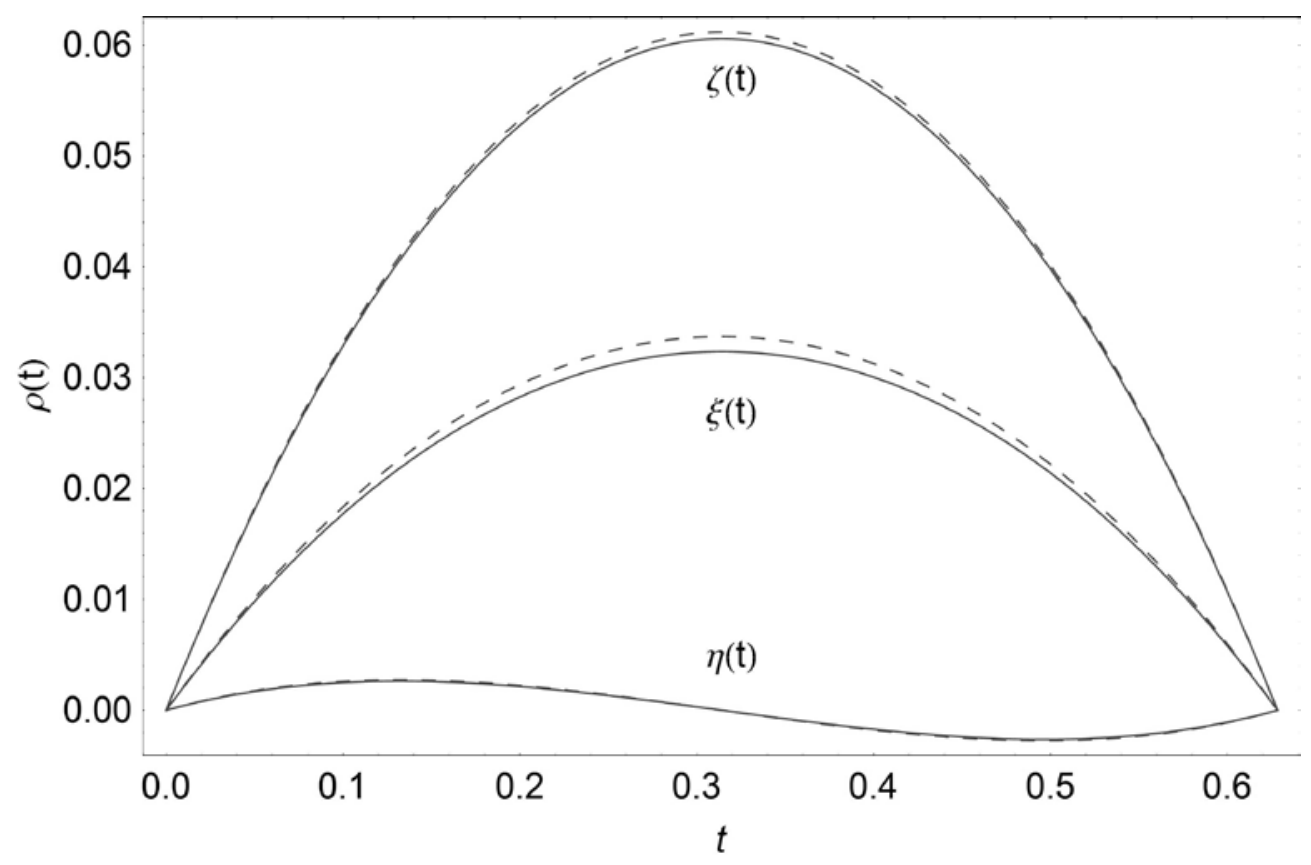


Figure 6.

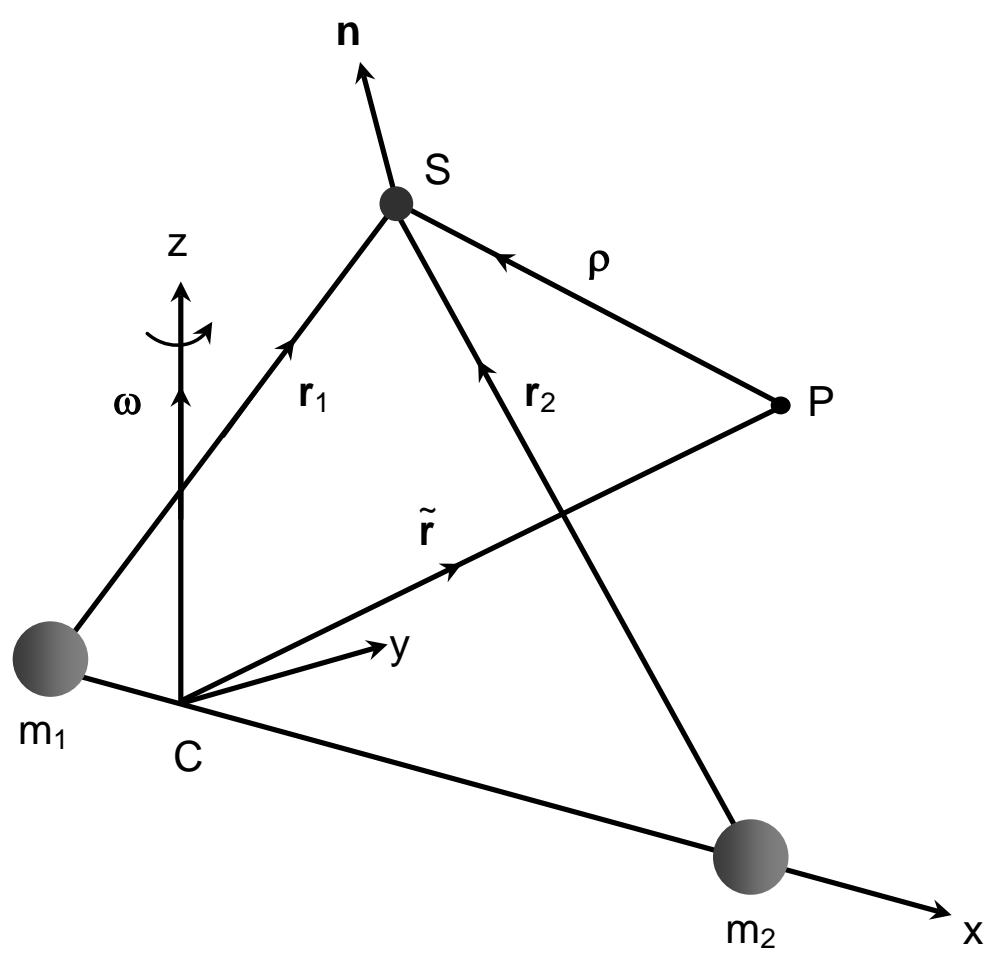


Figure 7.

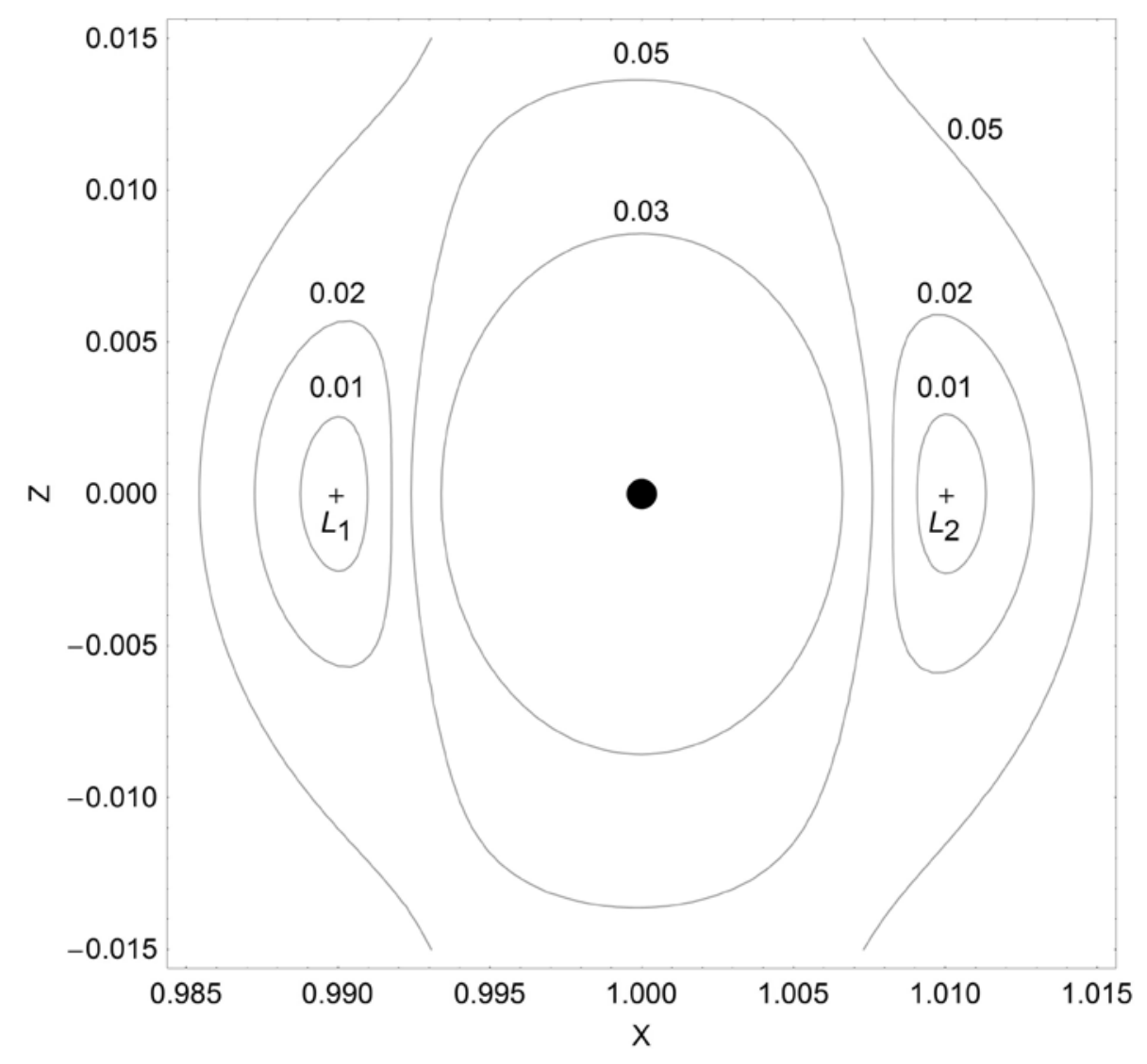


Figure 8.

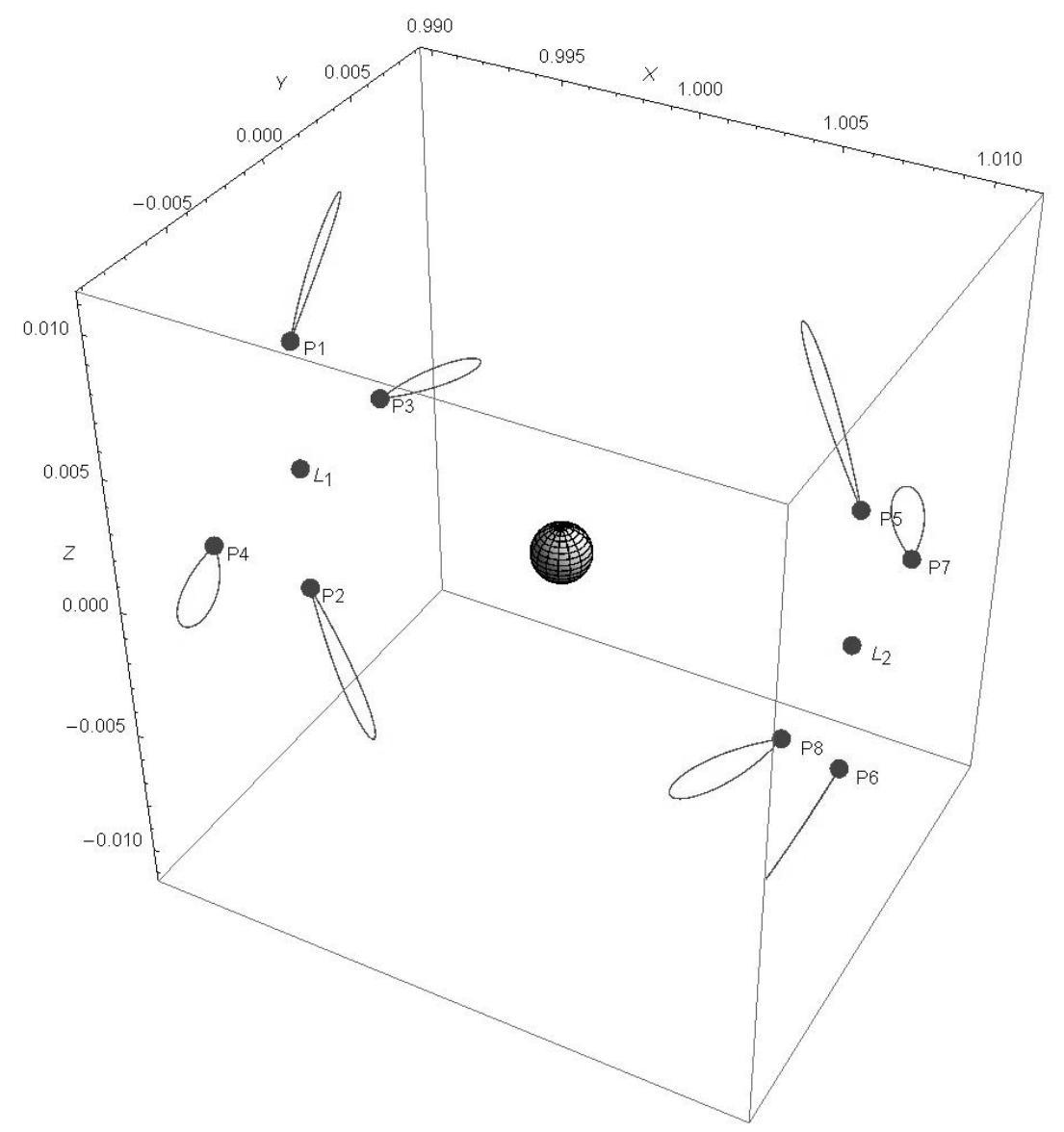


Figure 9.

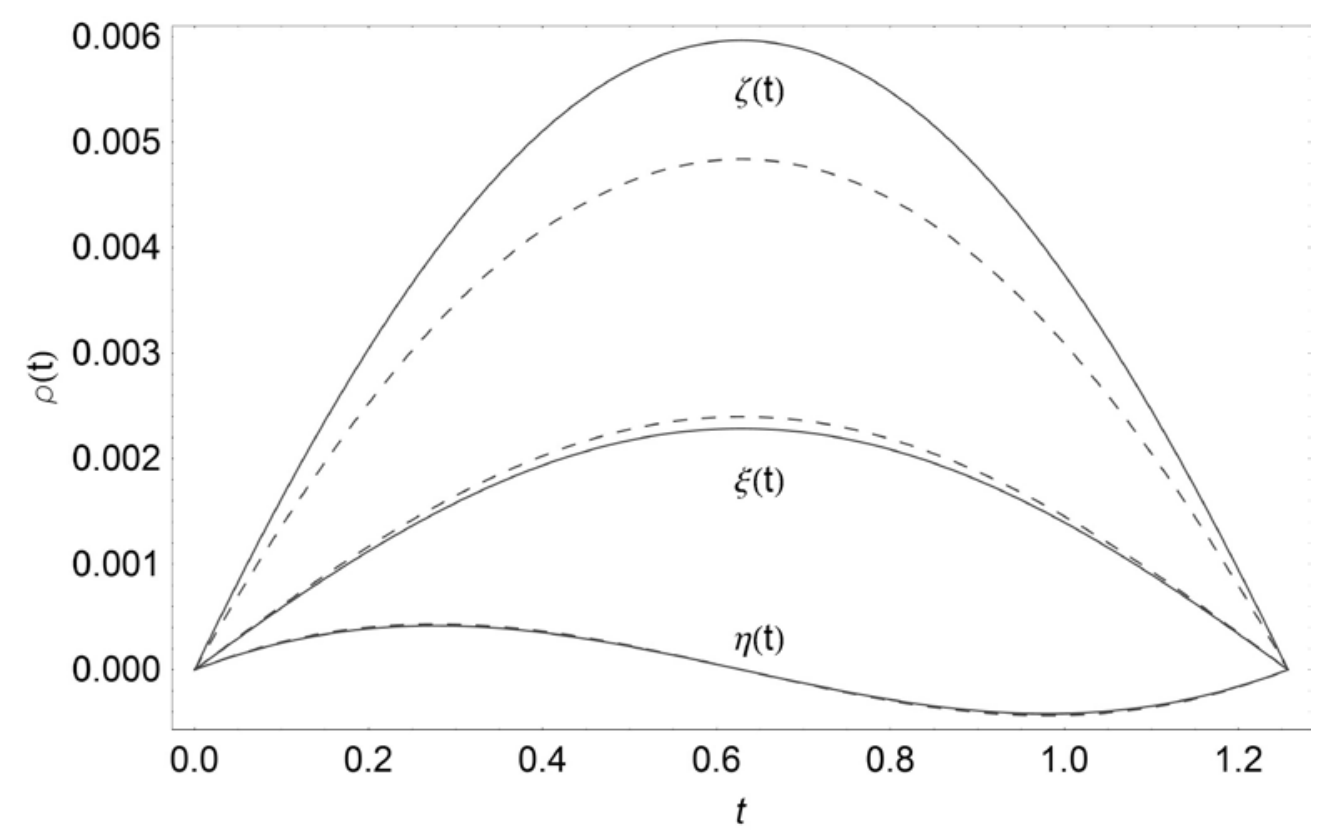


Figure 10.

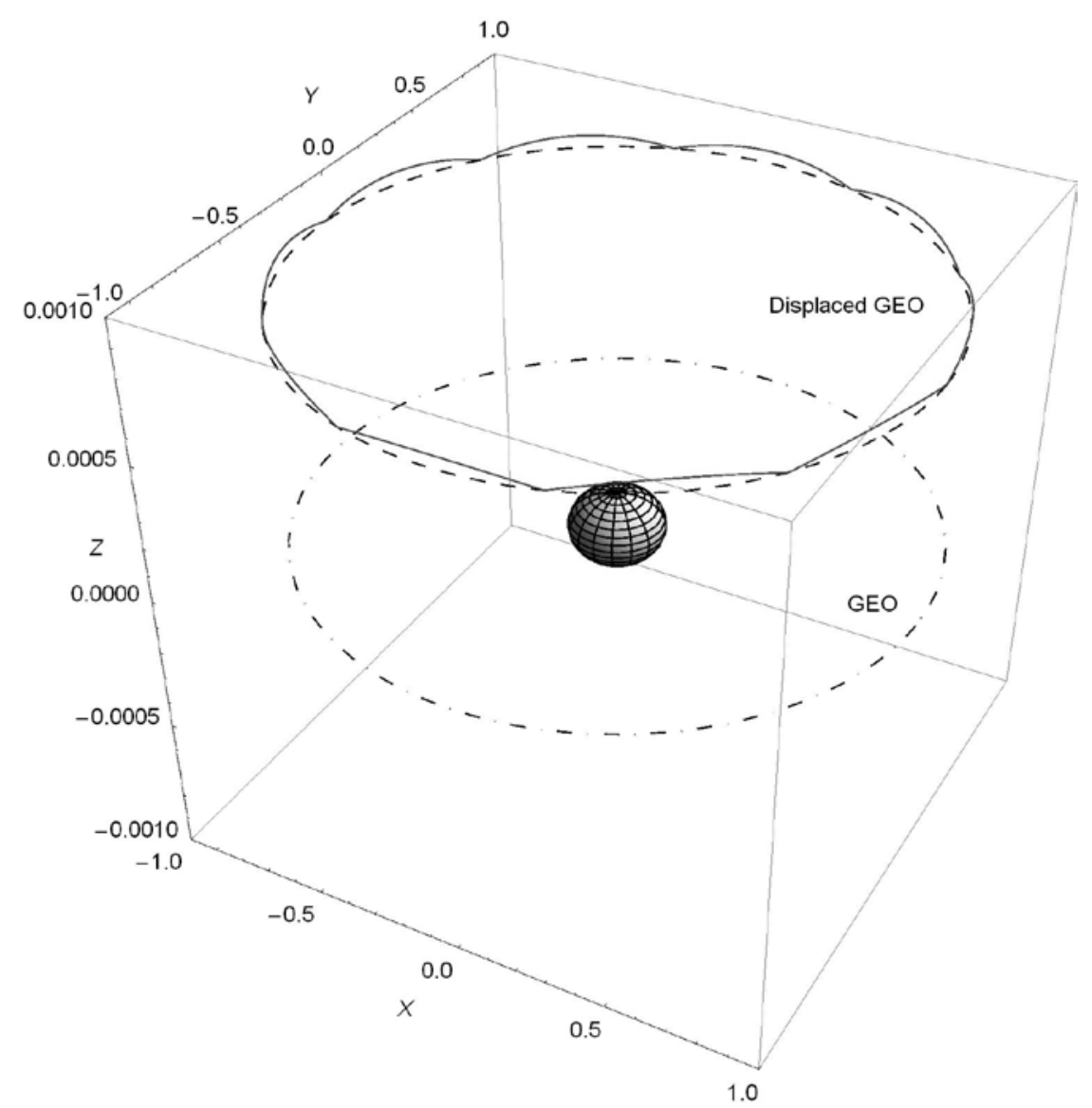

